\title{
The effectiveness and feasibility of fluid resuscitation directed by microcirculation monitoring in patients with septic shock: a randomized controlled trial
}

\author{
Qiuxiang Zhou ${ }^{1 \#}$, Chunyan Dai ${ }^{2 \#}$, Yan Zhu ${ }^{1}$, Tingting Han ${ }^{1}$, Jian Zhou ${ }^{1}$, Liang Zhao ${ }^{1}$ Xi Wang ${ }^{3}$, \\ Han Liu ${ }^{4}$, Jinlong $\mathrm{Qu}^{1}$, Wenfang $\mathrm{Li}^{1}$ \\ ${ }^{1}$ Department of Emergency and Critical Care, Shanghai Changzheng Hospital, Naval Military Medical University, Shanghai, China; ${ }^{2}$ Department \\ of Anesthesiology, Zhoupu Hospital, Shanghai Health Medical College, Shanghai, China; ${ }^{3}$ Department of Pediatrics, South Pier Community Health \\ Service Center, Shanghai, China; ${ }^{4}$ Department of Clinical Laboratory, Shanghai General Hospital Jiading Branch, Shanghai, China \\ Contributions: (I) Conception and design: Q Zhou, J Qu; (II) Administrative support: None; (III) Provision of study materials or patients: None; (IV) \\ Collection and assembly of data: Q Zhou, C Dai; (V) Data analysis and interpretation: H Liu, J Qu, W Li; (VI) Manuscript writing: All authors; (VII) \\ Final approval of manuscript: All authors. \\ \#These authors contributed equally to this work. \\ Correspondence to: Han Liu. Department of Clinical Laboratory, Shanghai General Hospital Jiading Branch, Shanghai 201803, China. \\ Email: liuhanhs@yeah.net; Jinlong Qu. Department of Emergency and Critical Care, Shanghai Changzheng 1. Hospital, Naval Military Medical \\ University, Shanghai 200003, China. Email: huanhun0513@163.com; Wenfang Li. Department of Emergency and Critical Care, Shanghai \\ Changzheng Hospital, Naval Military Medical University, Shanghai 200003, China. Email: chzhedlwf@163.com.
}

\begin{abstract}
Background: This study sought to examine fluid resuscitation in septic shock patients by monitoring their sublabial point of care microcirculation score (POEM) scores (a 3.5 cut-off value was used as the end point of recovery). It also sought to explore the effectiveness and safety of using the POEM score in the fluid resuscitation of septic shock.
\end{abstract}

Methods: Patients were randomly allocated to the experimental group or the control group. In the experimental group, a POEM score $>3$ was used as the end point of fluid resuscitation. In the control group, the doctor just monitor, don't know the data. Patients' heart rates, mean arterial pressure (MAP), Acute Physiology and Chronic Health Disease Classification System II (APACHE II) scores, Sequential Organ Failure Assessment (SOFA) scores, and oxygenation index scores were recorded at 2, 24, 48, $72 \mathrm{~h}$, and on the 7 th day after admission to the study. Statistically significant differences between the 2 groups were examined. Results: Thirty-one septic shock patients (comprising 14 patients in the experimental group and 17 patients in the control group) participated in our study. Patients' parameters upon admission to the study, including MAP, blood lactate and APACHE score, SOFA score, POEM score, cardiac output (CO), and central venous pressure (CVP), were recorded at $2 \mathrm{~h}$; There was no significant difference in the APACHE II scores, SOFA scores, and oxygenation index scores at $48 \mathrm{~h}$ between the 2 groups; however, at $72 \mathrm{~h}$, the scores of the experimental group were significantly better than those of the control group $(\mathrm{P}<0.05)$.

Conclusions: Under the guidance of POEM scores, limited fluid resuscitation reduced the intake of fluid any unnecessary amounts of fluids. POEM scores also offered certain protective effects to organ function at the early stage of septic shock, and did not affect patients' circulation.

Trial Registration: Chinese Clinical Trial Registry (ChiCTR2100049510).

Keywords: Fluid resuscitation; microcirulation; point of care microcirculation score (POEM); sepsis shock

Submitted Jun 22, 2021. Accepted for publication Aug 23, 2021.

doi: 10.21037/apm-21-1971

View this article at: https://dx.doi.org/10.21037/apm-21-1971 


\section{Introduction}

Septic shock is a common type of shock. It is classified as distributed shock, and is mainly characterized by the redistribution of fluid in the body with or without low cardiac output (CO). Microcirculation disorders are an important feature of septic shock. Microcirculation disorders related to septic shock have been confirmed by many studies $(1,2)$. In patients with septic shock, if macrohemodynamics does not change significantly, microcirculation will begin the process of changing from quantity changes to a quality change. Many studies have confirmed that the improvement of microcirculation appears to be related to the improvement of clinical prognosis $(3,4)$. Fluid resuscitation is a commonly used method to improve the hemodynamics of shock patients. It is very important that patients with septic shock are treated early. At present, the indicators for evaluating the effects of fluid resuscitation [e.g., macrohemodynamic indicators, such as mean arterial pressure (MAP), systolic blood pressure, heart rate, and central venous pressure (CVP), and metabolic indicators, such as blood lactic acid, tissue carbon dioxide $\left(\mathrm{CO}_{2}\right)$ ], lack reliability, may be invasive, lag behind the pathophysiological changes, cannot reflect the essence of shock changes in microcirculation, or have certain limitations (5-7).

Visualized microcirculation monitoring represents a new, reliable and intuitive method for evaluating the effects of fluid resuscitation. Previous studies have shown that microcirculation monitoring has good sensitivity and specificity in predicting the prognosis of septic shock. To date, very few studies have been conducted on the use of microcirculation as an indicator of fluid resuscitation. Indeed, studies have mainly used the percentage of perfused vessels (PPV) and microvascular flow index (MFI) to evaluate the effects of fluid resuscitation. PPV and MFI are mainly used to monitor microcirculation at a single location for a certain period of time. Compared to the POEM score, the score error of the one image is larger. Thus, theoretically, a POEM score should be more accurate at reflecting the changes of microcirculation than the PPV and MFI values at a single part. Previous studies showed that a POEM score of 3.5 is the best cut-off value for the prognosis of sepsis (8). This study used a POEM score of 3.5 as the resuscitation target or cut-off point to guide clinical fluid resuscitation for patients with septic shock. It was hoped that a new method based on the body's microcirculation would be found for fluid resuscitation in patients with septic shock.

We present the following article in accordance with the CONSORT reporting checklist (available at https://dx.doi. org/10.21037/apm-21-1971).

\section{Methods}

\section{Research subjects}

This is a two-parallel, single-blinded trial conducted in Shanghai Changzheng hospital. With the full informed consent of the patients or their families, patients who had been diagnosed with septic shock at the emergency department and intensive care unit (ICU) ward of Shanghai Changzheng hospital from September 2017 to January 2018 were enrolled in this study. The patients were allocated into the experimental group or the control group using simple randomization. In relation to the experimental group, if a patient had a POEM score greater than 3.5 points, the amount of fluid to be resuscitated was relatively controlled. To ensure safety, the fluid limit was based on the doctors' experience and rehydration was performed as necessary. If a patient had a POEM score less than 3.5 points, the treatment was administered based on the experience of the doctors in the previous trial group. Until a patient had a POEM score $>3$ points, their fluid infusion was restricted (as described above). In relation to the control group, the above indicators were only monitored, and the doctors were not notified of the results of patients in the experimental group. The fluid resuscitation of the control group was carried out based on the experience of the doctors in the experimental group. Patients' POEM scores were obtained at 2, 24, 48, $72 \mathrm{~h}$, and on the 7 th day. Patients' Acute Physiology and Chronic Health Disease Classification System II (APACHE II) scores, SOFA scores, oxygenation index scores, and other indicators were statistically diagnosed, and patients' heart rate, blood pressure (MAP), and blood lactic acid levels were recorded at the same time, $24 \mathrm{~h}$ fluid intake and output volume and so on, analysis of the data using statistics. The study was approved by Shanghai Changzheng Hospital [No. CZEC(2017)-09] and written informed consent was obtained from all patients. All procedures performed in this study involving human participants were in accordance with the Declaration of Helsinki (as revised in 2013).

\section{Selection criteria}

According to the 2016 Surviving Sepsis Campaign (SSC) 
guidelines on sepsis, Selection criteria include patients diagnosed with septic shock $(9,10)$ are patients with septic shock. Septic shock refers to infections with circulatory failure and abnormal tissue cell metabolism, which require vasoactive drugs to maintain blood pressure after fluid resuscitation.

\section{Exclusion criteria}

Patients were excluded if they were pregnant, had oral diseases, obvious mouth trauma, or bleeding, could not cooperate with the monitoring, required non-invasive ventilation, could not tolerate out off the ventilator effects, or had poor quality images that could not be analyzed.

\section{Experimental materials}

The GEM3000 blood gas analyzer produced by the American IL company was used. It was mainly used for to perform blood gas analyses of indicators, such as blood lactate values and oxygen partial pressure. The MicroVision microcirculation monitor was used to collect and analyze microcirculation images. Ultrasounds were used to monitor CO. The ultrasonic cardiac output monitor (USCOM) produced by Uscom, an Australian cardiovascular medical technology company, was used to monitor $\mathrm{CO}(\mathrm{L} / \mathrm{min})$.

\section{General information and data collection}

The following date was collected: (I) general information about patients' gender, age, diagnosis, site of infection, and prognosis at 28 days; (II) patients' organ function and metabolism of septic shock at 2, 24, 48, $72 \mathrm{~h}$, and on the 7 th day, including measurements of blood lactic acid, APACHE II scores, SOFA scores, oxygenation index scores, POEM scores, and CO; (III) $24 \mathrm{~h}$ fluid intake and output volume on the 1 st, 2 nd, $3 \mathrm{rd}$, and 7 th day.

\section{Other important indicators}

Acquisition of sublip microcirculation images: a trained operator used $37^{\circ} \mathrm{C}$ saline gauze or cotton swabs to gently wipe off secretions of the mouth and the upper lip. The probe was then placed on both sides of the patient's upper lip to collect 2 points on each side. Four images and POEM scores were obtained from the analysis of the microcirculation images by trained physicians. Data of patients' circulatory parameters were also collected.
CVP was measured by the internal jugular vein pressure transmitter. CO was measured by the echocardiographic output monitor at the suprasternal fossa and the aortic valve.

\section{Statistical methods}

This study used IBM SPSS Statistics 22 for the statistical analysis. Normally distributed measurement data are represented as mean \pm standard deviation $(\bar{x} \pm s)$, and 2 independent sample $t$-tests were used. Measurement data that did not conform to a normal distribution are represented as a median (M) [P25, P75], and MannWhitney U non-parametric tests were used. The count data are expressed as frequency (percentage) n (\%). A $\mathrm{P}<0.05$ indicated that the difference was statistically significant.

\section{Results}

Thirty-one patients (comprising 22 males and 9 females) suffering from septic shock who were admitted to our hospital from September 2017 to January 2018 were enrolled in the study. Written consent was obtained from the patients or their family members. The patients were randomly allocated to 2 groups. The experimental group comprised 14 patients ( 9 males and 5 females). The control group comprised 17 patients (13 males and 4 females). The allocation ratio is closed to 1:1 .Patients had the following primary diseases: 16 had severe pneumonia, 5 had abdominal infections (including acute gastrointestinal infection and spontaneous peritonitis), 6 had pancreatitis, 1 had diabetes complicated with skin and soft tissue infection, 1 had a lower extremity arterial embolism and infection, 2 had wound infections after spinal surgery. Of the 31 patients, 28 survived and 3 died (Figure 1).

\section{The situation and prognosis of the 2 groups of patients at the time of enrollment}

There was no statistically significant difference in patients' MAP, blood lactate levels, APACHE II scores, SOFA scores, POEM scores, CO, CVP or other measurements between the experimental group and the control group at 2 hours $(\mathrm{P}>0.05)$, nor was there any statistical difference on the 28th day of prognosis between the 2 groups (Table 1).

The statistics of relevant indicators on the 1st, 2 nd, and 


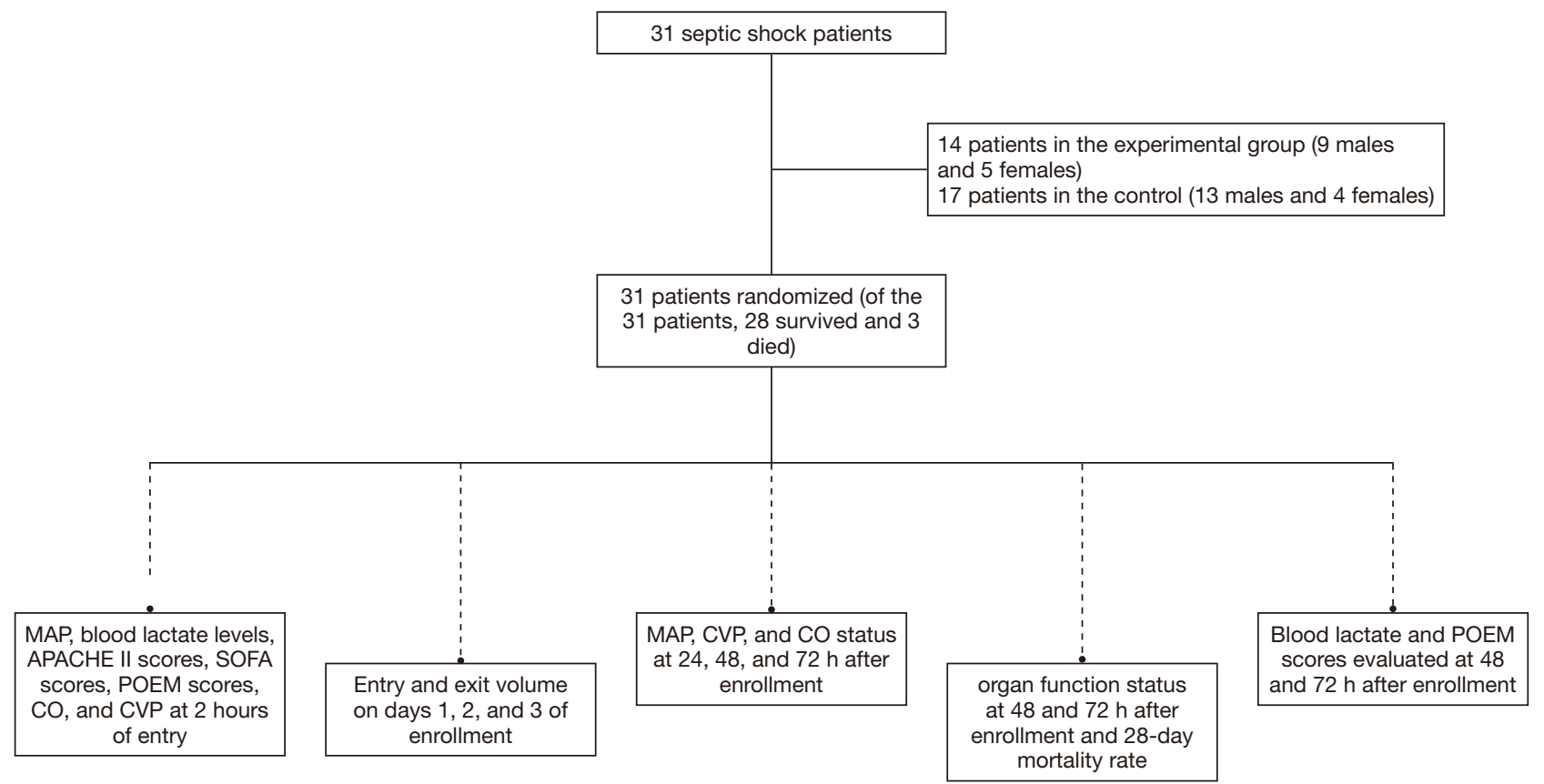

Figure 1 Group design scheme of septic shock patients. MAP, mean arterial pressure; SOFA, Sequential Organ Failure Assessment; POEM, Patient-Oriented Eczema Measure; CVP, central venous pressure; CO, cardiac output.

Table 1 General information of patients in the experimental and control groups

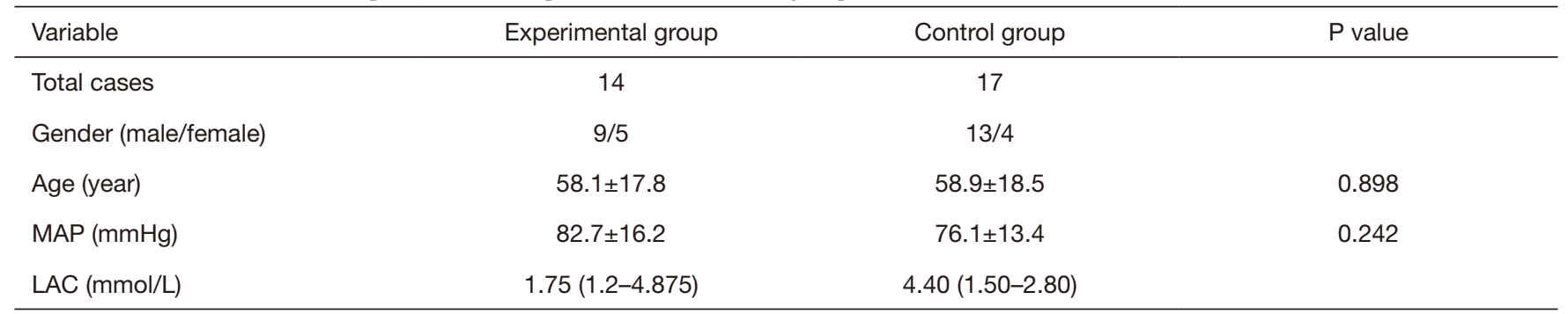

MAP, mean arterial pressure.

$3 r d$ days for patients after enrollment (the output includes $500 \mathrm{~mL}$ of water loss, including loss from the skin and breatbing) (Table 2)

As the results show, the amount of fluid intake in the experimental group on days 1 and 2 was significantly lower than that of the control group $(\mathrm{P}<0.05)$; however, there was no statistically significant difference in terms of output. There was no significant abnormality in the $24 \mathrm{~h}$ input and output on day 3. Thus, the fluid resuscitation of the control group mainly occurred in the first 2 days of diagnosis (Table 3).

The circulation of patients was evaluated at 24,48 , and
$72 \mathrm{~h}$. Most patients maintained a MAP and CO within a certain range through vasoactive drugs. In this study, regardless of whether certain fluid resuscitation was performed. There was no statistical difference between the 2 groups in terms of CO, MAP, and CVP $(\mathrm{P}>0.05)$. Regardless of whether fluid resuscitation was performed in the treatment group, certain methods were used to maintain a certain degree of hemodynamic stability. In this study, most of experimental group were maintained by norepinephrine (Table 4).

This study mainly used the APACHE II score, SOFA score and oxygenation index score to evaluate organ function. The results showed that there was no statistical 
Table 2 Entry and exit volume on days 1,2, and 3 of enrollment

\begin{tabular}{|c|c|c|c|c|c|c|}
\hline Variable & \multicolumn{2}{|c|}{ 1st day (24 h) } & \multicolumn{2}{|c|}{ 2nd day (24 h) } & \multicolumn{2}{|c|}{ 3rd day (24 h) } \\
\hline Experimental group & $3,090.6 \pm 855.2$ & $2,869.2 \pm 1,587.9$ & $2,343.6 \pm 997.4$ & $3,055.4 \pm 1125.3$ & $2,206.4 \pm 1,008.4$ & $2,880.0 \pm 1,323.1$ \\
\hline Control group & $4,002.8 \pm 879.5$ & $2,488.8 \pm 827.4$ & $3,470.1 \pm 619.1$ & $2,515.0 \pm 412.6$ & $2,869.6 \pm 909.6$ & $2,640.0 \pm 858.6$ \\
\hline$P$ value & 0.008 & 0.402 & 0.001 & 0.086 & 0.085 & 0.574 \\
\hline
\end{tabular}

Table 3 MAP, CVP, and CO status at 24, 48, and $72 \mathrm{~h}$ after enrollment

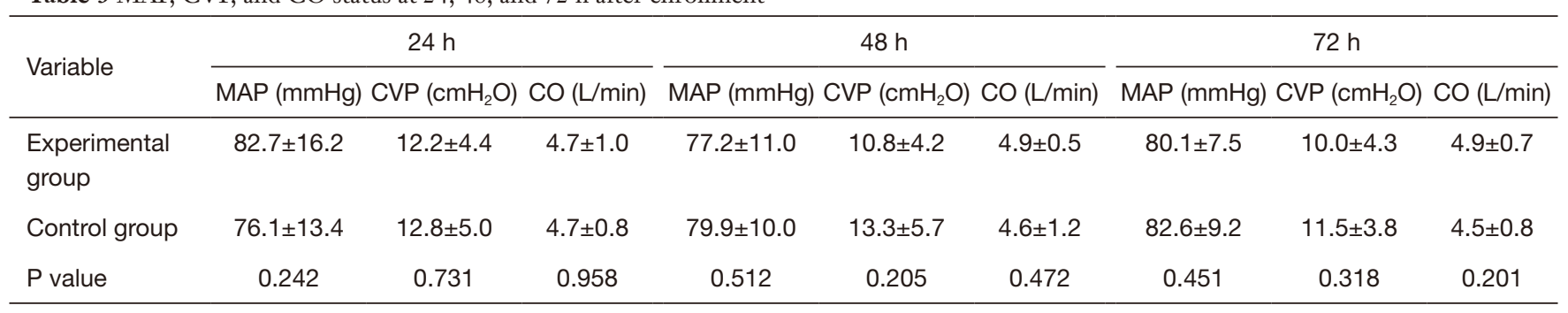

MAP, mean arterial pressure; CVP, central venous pressure; CO, cardiac output.

Table 4 Patients' organ function status at 48 and $72 \mathrm{~h}$ after enrollment and 28-day mortality rate

\begin{tabular}{|c|c|c|c|c|c|c|c|}
\hline Variable & \multicolumn{3}{|c|}{$48 \mathrm{~h}$} & \multicolumn{3}{|c|}{$72 \mathrm{~h}$} & $\begin{array}{c}28 \text { days } \\
\text { Mortality rate }\end{array}$ \\
\hline $\begin{array}{l}\text { Experimental } \\
\text { group }\end{array}$ & $10.0 \pm 5.1$ & $6.2 \pm 2.6$ & $201.7 \pm 61.1$ & $7.8 \pm 5.3$ & $4.9 \pm 2.8$ & $261.5 \pm 62.2$ & 7.6 \\
\hline Control group & $14.1 \pm 5.5$ & $8.3 \pm 3.1$ & $173.5 \pm 110.8$ & $11.9 \pm 3.1$ & $7.1 \pm 2.7$ & $205.5 \pm 70.6$ & 13.3 \\
\hline
\end{tabular}

SOFA, Sequential Organ Failure Assessment.

difference in APACHE II scores, SOFA scores, and oxygenation index scores between the 2 groups at 48 or at $72 \mathrm{~h}$ The index has all improved, but the primary outcomes are that, the experimental group improved more than the control group, and the difference between the 2 groups was statistically significant $(\mathrm{P}<0.05)$. There was no significant difference in the 28-day mortality rate between the 2 groups $(\mathrm{P}=0.575)$ (Table 5).

The experimental group underwent fluid control; however, it did not affect the perfusion of their tissues and organs. There was no significant difference in patients' blood lactic acid levels at 48 and $72 \mathrm{~h}$, and there was no significant difference in patients' blood flow and heterogeneity of microcirculation. Additionally, there was no statistically significant difference in patients' POEM scores $(\mathrm{P}>0.05)$, indicating that the control of resuscitation fluid under the guidance of POEM scores did not affect the perfusion of tissues and organs.

\section{Discussion}

Sepsis has a high mortality rate. If it is not treated in time, it can progress to septic shock, effective blood volume reduction, insufficient tissue and organ perfusion, and then progress to sepsis-related multiple organ dysfunction syndrome. Early improper treatment leads to reduced oxygen delivery and anaerobic metabolism. The increase and early correction of tissue and organ hypoperfusion is an important measure in improving prognosis (10), and it is also an important goal of early cluster therapy. Clinical findings indicate that when macrocirculation stabilizes, the pathological changes related to the microcirculation 
Table 5 Blood lactate and POEM scores evaluated at 48 and $72 \mathrm{~h}$ after enrollment

\begin{tabular}{lccccc}
\hline \multirow{2}{*}{ Variable } & \multicolumn{2}{c}{$48 \mathrm{~h}$} & & \multicolumn{2}{c}{$72 \mathrm{~h}$} \\
\cline { 2 - 3 } \cline { 5 - 6 } & Lac $(\mathrm{mmol} / \mathrm{L})$ & POEM score $(\mathrm{score})$ & Lac $(\mathrm{mmol} / \mathrm{L})$ & POEM score $(\mathrm{score})$ \\
\hline Experimental group & $1.60(1.375,2.85)$ & $3.6 \pm 0.7$ & $1.60(1.300,2.975)$ & $3.8 \pm 0.4$ \\
Control group & $2.50(2.20,3.20)$ & $3.4 \pm 0.9$ & $1.95(1.80,3.825)$ & $3.7 \pm 0.5$ \\
P value & 0.085 & 0.063 & 0.054 & 0.553 \\
\hline
\end{tabular}

still exist, and tissue perfusion does not improve (11). Improvements related to macrocirculation fail to meet the needs for tissue metabolism. The evaluation of tissue metabolism in patients with sepsis and septic shock is of great significance.

Fluid resuscitation is an important treatment step in the treatment of sepsis. The theory is based on hemodynamic changes in patients with sepsis or septic shock. Effective reduced vascular content and decreased vascular tension due to an inflammatory factor cause continuous deterioration in hemodynamics (12). The supplementation of volume can increase the effective circulating vascular volume to a certain extent, and increase oxygen delivery to improve the function of microcirculation $(13,14)$. However, a large amount of fluid resuscitation also has certain side effects, such as tissue edema (15) and electrolyte abnormalities. In relation to the actual operation of fluid resuscitation, there is currently no unified evaluation index that indicates how much fluid is appropriate. In their milestone study of fluid resuscitation, Rivers et al. (10) developed a fluid resuscitation strategy that targets early goal-directed therapy (EGDT). Provisions include indicators such as CVP, MAP, urine volume as the end point of resuscitation. During the 6 hours before resuscitation, patients in the EGDT group received more fluid (5.0 to $3.5 \mathrm{~L}, \mathrm{P}<0.01)$, higher transfusion rate $(\mathrm{P}<0.01)$, more dopamine butamine (13.7\% to $0.8 \%)$, but lower mortality $(\mathrm{P}=0.009)$. Several large multi-center studies have also confirmed the benefits of EGDT (e.g., ProCESS, ARISE, and ProMISe). However, many questions about EGDT remain, including whether it can be used as a routine treatment in fluid infusion.

There is a great deal of difference between the amount of fluid in a standardized treatment and others, and the EGDT group is only the 6-hour treatment goal. Whether a fluid resuscitation strategy that targets early goaldirected therapy (EGDT) can explain many questions about EGDT is also questionable. The CVP-mediated fluid resuscitation strategy requires CVP to reach 8-
$12 \mathrm{mmHg}$, blood pressure to reach $65 \mathrm{mmHg}$ or more, and central blood oxygen saturation $(\mathrm{ScvO} 2)$ to reach $70 \%$ or more within 6 hours; however, it is difficult to implement clinically. The operation may require more solution, which produces more side effects. Many multi-center studies have confirmed that EGDT does not have more advantages than empiric treatments for fluid resuscitation, and has more complications $(16,17)$. In the FEAST study $(18)$, it was found that early bolus fluid infusion increased the mortality rate at $48 \mathrm{~h}$ (the experimental group $10.5 \%$ vs. the control group $7.5 \%$ ), and that too much fluid was harmful to each subgroup.

Fluid infusion can improve shock. However, studies have confirmed that when CVP is higher than $12 \mathrm{mmHg}$, MFI and PPVs decrease significantly, which suggests that high CVP is not conducive to the recovery of microcirculation (19). Thus, the question as to what a better indicator is for evaluating fluid resuscitation arises. Returning to the innate character of the shock is the obstacle of microcirculation. Microcirculation is the production place in which tissues obtain oxygen and nutrients. It is also the place in which metabolites and $\mathrm{CO} 2$ are excluded. Thus, for fluid resuscitation during the shock, microcirculation recovery is critical. Lactic acid monitoring may be a useful indicator of microcirculation recovery. The reduction of lactic acid to normal may be an indicator of the improvement of septic tissue perfusion and oxygenation. Jansen et al. conducted a normal multi-center randomized control trial (RCT) to Lactic acid levels reduced hospital mortality (7). Lactic acid is indeed a good indicator of tissue perfusion, but lactic acid needs to constantly draw the patient's blood, and continuous clinical dynamic monitoring is limited. Further, abnormal liver function affects the lactic acid clearance rate, and the guiding value of lactic acid monitoring needs to be carefully considered.

Research has shown that (8) the POEM score is positively correlated with blood lactate, and a POEM score of 3.5 on the day of diagnosis can be used as an evaluation 
index for the prognosis of sepsis. Thus, in this study, under the guidance of the POEM score, a fluid resuscitation strategy was used to guide the exploration of new methods of fluid resuscitation. The POEM scores of patients in the experimental group were monitored. For patients with a POEM score $>3$, the fluid resuscitation volume was relatively controlled. If blood pressure was low, it was maintained by vasoactive drugs. In the control group, fluid resuscitation was performed based on doctors' treatment experience. The results showed that there was no significant difference in patients' MAP, blood lactate levels, APACHE II scores, SOFA scores, POEM scores, CO, and CVP between the experimental group and the control group at 2 hours of entry $(\mathrm{P}>0.05)$. On the 1st and $2 \mathrm{nd}$ day, the intake of the experimental group was significantly lower than that of the control group, suggesting that the fluid resuscitation of the control group was mainly concentrated to the first 2 days of diagnosis. Patients' circulation was evaluated at 24, 48, and $72 \mathrm{~h}$. Most patients maintained MAP and $\mathrm{CO}$ within a certain target range using vasoactive drugs. There was no statistical difference between the 2 groups in terms of CO, MAP, and CVP. Specifically, there was no statistical difference in patients' APACHE II scores, SOFA scores and oxygenation index scores between the 2 groups at $48 \mathrm{~h}$. At $72 \mathrm{~h}$, the APACHE II scores, SOFA scores, and oxygenation index scores of patients in the experimental group were better than those of patients in the control group, suggesting that restrictive fluid resuscitation may be effective at protecting early organ function, and that resuscitation with too much fluid is not very beneficial at maintaining MAP and CO. Further, it may increase tissue edema or overload the heart and cause patients' conditions to worsen.

We also found that there was no significant difference in patients' blood lactic acid levels at 48 and $72 \mathrm{~h}$, and no significant difference in patients' blood flow and the heterogeneity of microcirculation. Further, there was no significant statistical difference in patients' POEM scores $(\mathrm{P}>0.05)$. Thus, POEM scores can be used to guide the treatment of fluid resuscitation in sepsis. POEM scores have the same guiding significance as lactic acid in the assessment of tissue perfusion after fluid resuscitation in sepsis or septic shock. Additionally, the POEM score is obtained in a non-invasive manner and enables continuous dynamic monitoring. Thus, POEM scores can be used to guide restrictive fluid resuscitation treatment early in time, save time and costs, and reduce possible errors in clinical lactate monitoring.

This study showed that restrictive fluid resuscitation guided by POEM scores can reduce the amount of unnecessary early resuscitation fluid, has a certain protective effect on organ function in the early stage of septic shock, and does not affect patients' circulation. However, this study had a number of limitations. For example, the sample size was still relatively small. Further, when a POEM score of a patient in the experimental group was greater than 3 points, the question of how to accurately control the fluid resuscitation volume was still made based on experience. Further research needs to be conducted to increase the sample size to achieve more specific fluid control.

\section{Acknowledgments}

Funding: Shanghai Municipal Science and Technology Commission of the Yangtze River Delta Cooperation Key Project (18411951400, 19495810200, 14495810400): Research and Development and Demonstration Application of Key Technologies for Emergency Rescue in the Yangtze River Delta based on Real-Time Detection and Rapid Injury Assessment (14495810400). Study on the Early Identification and Prevention of Sepsis (18411951400), shanghai municipal health commission project (gwv-10.1-xk24).

\section{Footnote}

Reporting Checklist: The authors have completed the CONSORT reporting checklist. Available at https://dx.doi. org/10.21037/apm-21-1971

Trial Protocol: Available at https://dx.doi.org/10.21037/apm21-1971

Data Sharing Statement: Available at https://dx.doi. org/10.21037/apm-21-1971

Conflicts of Interest: All authors have completed the ICMJE uniform disclosure form (available at https://dx.doi. org/10.21037/apm-21-1971). The authors have no conflicts of interest to declare.

Ethical Statement: The authors are accountable for all aspects of the work in ensuring that questions related 
to the accuracy or integrity of any part of the work are appropriately investigated and resolved. The study was approved by Shanghai Changzheng Hospital [No. CZEC(2017)-09] and written informed consent was obtained from all patients. All procedures performed in this study involving human participants were in accordance with the Declaration of Helsinki (as revised in 2013).

Open Access Statement: This is an Open Access article distributed in accordance with the Creative Commons Attribution-NonCommercial-NoDerivs 4.0 International License (CC BY-NC-ND 4.0), which permits the noncommercial replication and distribution of the article with the strict proviso that no changes or edits are made and the original work is properly cited (including links to both the formal publication through the relevant DOI and the license). See: https://creativecommons.org/licenses/by-ncnd/4.0/.

\section{References}

1. Goedhart PT, Khalilzada M, Bezemer R, et al. Sidestream Dark Field (SDF) imaging: a novel stroboscopic LED ring-based imaging modality for clinical assessment of the microcirculation. Opt Express 2007;15:15101-14.

2. Groner W, Winkelman JW, Harris AG, et al. Orthogonal polarization spectral imaging: a new method for study of the microcirculation. Nat Med 1999;5:1209-12.

3. De Backer D, Donadello K, Sakr Y, et al. Microcirculatory alterations in patients with severe sepsis: impact of time of assessment and relationship with outcome. Crit Care Med 2013;41:791-9.

4. Tachon G, Harrois A, Tanaka S, et al. Microcirculatory alterations in traumatic hemorrhagic shock. Crit Care Med 2014;42:1433-41.

5. Meyer J, Shankar-Hari M. Protocolised early goaldirected therapy in patients with sepsis/septic shock does not result in improved survival compared with usual care with less invasive resuscitation strategies. Evid Based Med 2017;22:223.

6. Bakker J, Nijsten MW, Jansen TC. Clinical use of lactate monitoring in critically ill patients. Ann Intensive Care 2013;3:12.

7. Jansen TC, van Bommel J, Schoonderbeek FJ, et al. Early lactate-guided therapy in intensive care unit patients: a multicenter, open-label, randomized controlled trial. Am J Respir Crit Care Med 2010;182:752-61.

8. Qu J, Zhang S, Han T, et al. First day POEM scores predict outcomes for septic shock patients: a retrospective observational study. Int J Clin Exp Med 2019;12:12250-6.

9. Rhodes A, Evans LE, Alhazzani W, et al. Surviving Sepsis Campaign: International Guidelines for Management of Sepsis and Septic Shock: 2016. Intensive Care Med 2017;43:304-77.

10. Rivers E, Nguyen B, Havstad S, et al. Early goal-directed therapy in the treatment of severe sepsis and septic shock. N Engl J Med 2001;345:1368-77.

11. De Backer D, Creteur J, Preiser JC, et al. Microvascular blood flow is altered in patients with sepsis. Am J Respir Crit Care Med 2002;166:98-104.

12. Suffredini AF, Fromm RE, Parker MM, et al. The cardiovascular response of normal humans to the administration of endotoxin. $\mathrm{N}$ Engl J Med 1989;321:280-7.

13. Singel DJ, Stamler JS. Chemical physiology of blood flow regulation by red blood cells: the role of nitric oxide and S-nitrosohemoglobin. Annu Rev Physiol 2005;67:99-145.

14. Anning PB, Finney SJ, Singh S, et al. Fluids reverse the early lipopolysaccharide-induced albumin leakage in rodent mesenteric venules. Intensive Care Med 2004;30:1944-9.

15. Woodcock TM, Woodcock TE. Revised Starling equation predicts pulmonary edema formation during fluid loading in the critically ill with presumed hypovolemia. Crit Care Med 2012;40:2741-2; author reply 2742 .

16. ARISE Investigators; ANZICS Clinical Trials Group; Peake SL, et al. Goal-directed resuscitation for patients with early septic shock. N Engl J Med 2014;371:1496-506.

17. Rusconi AM, Bossi I, Lampard JG, et al. Early goaldirected therapy vs usual care in the treatment of severe sepsis and septic shock: a systematic review and metaanalysis. Intern Emerg Med 2015;10:731-43.

18. Maitland K, Kiguli S, Opoka RO, et al. Mortality after fluid bolus in African children with severe infection. $\mathrm{N}$ Engl J Med 2011;364:2483-95.

19. Vellinga NA, Ince C, Boerma EC. Elevated central venous pressure is associated with impairment of 
microcirculatory blood flow in sepsis: a hypothesis generating post hoc analysis. BMC Anesthesiol 2013;13:17.

Cite this article as: Zhou Q, Dai C, Zhu Y, Han T, Zhou J, Zhao L, Wang X, Liu H, Qu J, Li W. The effectiveness and feasibility of fluid resuscitation directed by microcirculation monitoring in patients with septic shock: a randomized controlled trial. Ann Palliat Med 2021;10(8):9069-9077. doi: 10.21037/apm-21-1971
(English Language Editor: L. Huleatt) 